\title{
Metrology for the protection of individuals and workers in the field of radiation
}

\section{La métrologie au service de la protection des particuliers et des travailleurs dans le domaine des rayonnements}

\author{
François SUDER ${ }^{1}$ et Sophie VERHAEGHE ${ }^{2}$ \\ ${ }^{1}$ COFRAC Section Laboratoires Pôle Physique-Mécanique 52 rue Jacques Hillairet 75012 PARIS France \\ ${ }^{2}$ COFRAC Section Laboratoires Pôle Bâtiment-Electricité 52 rue Jacques Hillairet 75012 PARIS France
}

Key Words / Mots Clés: regulation scheme, accreditation, protection, radiation / schéma réglementaire, accreditation, protection, rayonnements

- Abstract. The authors will offer to present the purpose of metrology in the regulation scheme to protect the individuals and workers in the field of radiations, from ionizing radiation to electro-magnetic ones. Calibration ways and measurement methods will be introduced for three types of radiations: ionising radiation, visible radiation and electromagnetic radiation. For each wavelength domain the authors will develop the cooperation between the regulation and the accreditation schemes to guarantee the quality of measurements, with some information about the specific metrology developed to assure traceability to the international system and some specific methods of measurement.

\section{Introduction}

To assure the protection of individuals and workers in the field of radiation, some accreditation and regular schemes had been developed. For each wavelength domain specific metrological aspects had to be solve to be able to assure traceability to the International System (SI). In these regular domains, accredited laboratories had to be able to give secured results with high level metrology.

Three wavelength domains will be developed in this article:

- Ionising radiation: worker dosimetry (external dosimetry and anthroporadiometry) with the connection between accreditation and regulation with specific traceability using consensus standards (anthropomorphic phantom) and specific measurement. Accreditation in environmental field is also available to give information to individuals including measurement of Radon radiation.

- optical radiation: traceability of UV radiometer used in measurement for the control of tanning booth in an inspection scheme or measurement rules in the frame of ROA (artificial optical radiation) regulation scheme or calibration of Elisa test plate for serology (allergen) measurement for food

- Electromagnetic radiation: SAR measurement and on-site radiation field measurement in regulatory and accreditation scheme

(C) The Authors, published by EDP Sciences. This is an open access article distributed under the terms of the Creative Commons Attribution 


\section{2. lonising radiations}

\subsection{Regular scheme}

To begin, in the field of ionising radiation, a regular scheme with an agreement treated by the French Nuclear Safety Authority (ASN) linked with an accreditation is presented. The approach allows an efficient following of the protection of workers. Sub-domains in this regular field are: external dosimetry (passive dosimetry), anthroporadiometry and radiotoxicology.

Radiotoxicology field is linked with Biomedical laboratories, accredited with the standard NF EN ISO 15189 and will not be developed in this article.

To follow the exposition of workers to the ionising radiation, the regular scheme asks for an accreditation. Measurement was done by accredited laboratories. An agreement was given by the ASN to give a legal value to these measurements. As usual in an accreditation scheme, periodical evaluation was done by the accreditation body following the standard NF EN ISO/CEI 17025. Regular aspects were checked by some ASN inspections. This combination of coupled evaluations is able to validate good measurements with correct accuracy and adapted metrology.

Worker dosimetry is done through personal equivalent dose measurements. These measurements need the use of anthropomorphic phantoms. So for anthroporadiometry measurement, anthropomorphic phantom are use for traceability to SI. These phantoms are well defined in some international publication. These phantoms are also used for interlaboratory comparisons, organised in the regular field or even in voluntary field to give guaranties in measurement results. Local phantoms can also be used. The results of measurements done with these phantoms are examined during periodical evaluation linked with interlaboratory comparisons done with some consensual phantoms.

On another hand, some parameters had been defined as non critical measurement and it allows laboratories to avoid a traceability scheme in compliance with accreditation rules. For example the temperature of the measurement room for anthroporadiometry had be considered as a non critical environmental parameter taking into account data from manufacturer of the sensor elements and the energy peak due to the radiation from the potassium ${ }^{40} \mathrm{~K}$ from bones. The energy of this peak is well known and any effect of temperature or sensor drift can easily be detected.

These specifics points in traceability scheme of measurement allow sufficient accuracy to have pertinent data for the following of the survey of the exposition of workers to ionising radiation.

\section{2 environmental scheme}

Some accreditations cover also measurement in environmental radionuclide research scheme in liquid, solid and gaseous matrix. A great number of laboratories accredited for these measurements cover the surface of our country.

These environmental measurements allow the following of the radiation and contamination applied on French individuals.

Some laboratories asked also to be accredited in Radon radiation measurements used in some geographic well defined places in France.

\section{Optical radiations}

In the field of optical radiations, some specific domains will be shortly developed with accreditations in regular schemes.

\subsection{Tanning booth}

To protect individuals, a regular scheme had been developed to check radiation issued from artificial ultra-violet radiation in tanning booth. Regulation well defined UV radiation limit in tanning booth. Accredited inspection organisms were mandated to perform these technical controls (initial and periodic). In the frame of these inspections, they need specific UV radiometer to perform measurements to check these tanning installations by artificial UV radiations using UV1 to UV3 apparatus. For these specific measurements, inspectors need specific instrument with a correct traceability to SI. Accredited laboratories had to develop specific calibration for these instruments in the complete wavelength range to be able to cover regular requirement for tanning booth control. Corresponding scope of accreditation for calibration of UV radiometer had to be extended.

Accredited calibration offer had to be improved to be able to answer to the requirement of the inspector in charge of the application of the control of the regulation. 
Traceable measurements for the control of tanning installations improve the protection of individuals against artificial UV radiation.

\subsection{Artificial optical radiation}

Following the directive $\mathrm{n}^{\circ} 2006 / 25 / \mathrm{CE}$ from European parliament, a new regulation scheme had been developed in France. This scheme asks for an accreditation for measurements in the field of artificial optical radiation (ROA). The requirements cover optical radiation from infrared radiation to ultra-violet including visible radiations. This double scheme (regulation and accreditation) is applicable for incoherent radiation and laser radiation.

The aim of this scheme is to have some accredited laboratories for measurement of level of exposition to ROA on working place.

Some metrological aspects have to be solved like the covering of the wavelength range (in one or more domains) and the equipment adapted for these measurements. Two types of instruments had been focused: radiometer and spetroradiometer. Equilibrium between the cost of the instruments and the number of measurement needed to cover all situations in the working place need a real reflexion taking into account metrological aspect including traceability to SI.

An exposition to UV radiation, infrared radiation or visible radiation could have different effect. But in all case, the evaluation of the risk for a worker shall have to be estimate and in some cases some measurement should be done in specific conditions representative of the work conditions. This evaluation of the risk and/or measurement must be compare with limits defined in regulation.

Specific metrology had to be taking into account depending of the choice of the instrument and methods of measurement linked with the regulation to protect workers against an exposition to ROA.

\section{3 food safety}

Some laboratories are accredited for measurement for food safety to look for some allergen in food. These laboratories used optical measurements (ELISA). These instruments have to give results traceable to SI. Some specific standards have to be calibrated for transmission quantities. Calibration equipments and procedures in calibration accredited laboratories had to be adapted to be able to calibrate standard in geometry similar to those used in ELISA instruments.

The quality of these measurements and the linked metrology allow high accurate evaluation of the data to protect individuals about different allergen in food.

\section{Electromagnetic field}

Nowadays, different accreditation programs exist, dealing with non-visible and non-ionising radiation in the electromagnetic spectrum. They are basically entitled to gather the following tests:

- electromagnetic emission and immunity

- telecommunications terminals equipments testing

- $\quad$ specific absorption rate (SAR) measurements

- electromagnetic fields measurement for human exposure

- $\quad$ on-site electromagnetic fields measurements

- radar fields measurement

All those types of electromagnetic tests are very useful for any consumer. Nonetheless, only one small part of those programs is written in a regular scheme: on-site electromagnetic fields measurements, close to transmitting stations.

Dealing with this program, accreditation has been made mandatory by the authority in charge of the regulation of the radiofrequencies in France, the ANFR (Frequencies National Agency). It means that anyone who wants to be publicly recognised to measure on-site radiofrequencies needs to be accredited as a testing laboratory by the Cofrac and, prior to this procedure, has to file a record with the ANFR.

The regulation is not only limited to mobile phone frequencies (GSM, UMTS, LTE), but also includes Wi-Fi wavelengths, Professional Mobile Radio (PMR) services or FM broadcasting. Furthermore, the last version of the 
measurement protocol created by the ANFR and mandatory for the accredited laboratories of the domain includes smart networked meters (Linky smart meters) working out at lower frequencies.

To perform this type of tests, laboratories must invest in some essential equipment, as spectrum analysers, antennas and field probes. Currently, other laboratories across the world are accredited for the calibration of these electrical devices, so that traceability to the International System can be proven anytime. Also, uncertainties linked to the measurement ought to be jotted down into the tests reports, so that metrology can be applied from the beginning to the end.

Considering the other programs, even if they are not linked to any regular scheme, they are essential for our modern society. Indeed, they allow controlling that the equipment sold in our countries respect the norms and don't harm the population. For instance, accrediting some laboratories for the SAR measurements allow to control that the tests performed on mobile phones or tablets accord with the rules defined for launching new products.

To perform SAR tests, laboratories used to buy phantoms filled with some liquid, to respond to the various phone frequencies as the human body or head uses to do. However, for those measurements, not only electrical skills are required, but also chemistry ones, because of the dielectric liquids employed. The control of the phantom is only a visual one, and the virtual network analyser or the measurement probes can be calibrated by accredited laboratories. Furthermore, each instrument is cautiously verified before any use, to perform the best tests anytime.

However, lately another type of measuring equipment has arrived on the market, simulating the human body without using all those interchangeable liquids. Indeed, the brand new phantom consists in a device using a vector probe-array technology able to measure any Device Under Test among a large range of frequencies. Even if the calibration of this type of device is still hard to perform as it is some multi-skill machinery, the possibilities offered by those devices are bright.

Finally, another program corresponding to a regular scheme has to be developed in accordance with the French General Labour Directorate, in order to protect not the individuals as described in the two previous programs, but the workers. Indeed, a law text has been published in August 2016 in France, stipulating that any firm employing people near transmitting stations must comply with electromagnetic fields limitations.

For the majority of companies, these guidelines don't affect the way employees are managed, but it changes everything for people working on rooftops, or close to antennas. Indeed, for those critical working spots, employers ought to protect their workers (particularly pregnant women, or teenage employees) not to be overexposed to environmental electromagnetic fields during their working shifts.

Nevertheless, even if the rule has already entered the French law, no accreditation program has been developed for the moment. This evolution could lead to a wave of new laboratories candidate for accreditation recognition.

\section{Conclusions}

In different wavelength domains, radiation could have an effect on the health of individuals and workers.

Regulation linked with some accreditation schemes work together to offer laboratories which are able to perform measurements of high accuracy and high level of traceability to SI to protect individuals and workers.

It is not possible to avoid all radiation in our environment but with adapted protections (individual or structural) and correct measurements, it's possible to know and limit the effects of this radiations.

The quality of the measurements and the linked metrology given by accredited laboratories in the frame of regular schemes is a good tool to protect individuals and workers. 